\title{
Functionalization of Carbon Spheres with a Porphyrin-Ferrocene Dyad
}

\author{
Fabio Possanza ${ }_{1}^{[a]}$ Francesca Limosani, ${ }^{[a]}$ Pietro Tagliatesta, ${ }^{*[a]}$ Robertino Zanoni, ${ }^{*[b]}$ \\ Manuela Scarselli, ${ }^{[c]}$ Erica Ciotta, ${ }^{[d]}$ and Roberto Pizzoferrato ${ }^{[\mathrm{d}]}$
}

\begin{abstract}
Meso-tetraphenylporphyrin connected with a ferrocene molecule in the beta-position of the macrocycle through a triple carbon-carbon bond has been bound to carbon spheres using the Prato-Maggini reaction. The ethynyl or/and phenylene ethynylene subunits were chosen as a linking bridge to give a high conjugation degree between the donor (i.e., ferrocene), the photoactive compound (i.e., porphyrin), and the acceptor (i.e., carbon spheres). The molecular bridges have been directly
\end{abstract}

linked to the beta-pyrrole positions of the porphyrin ring, generating a new example of a long-range donor-acceptor system. Steady-state fluorescence studies together with Raman and XPS measurements helped understanding the chemical and physical properties of the porphyrin ring in the new adduct. The spectroscopic characteristics were also compared with those obtained from a similar compound bearing fullerene instead of carbon spheres.

\section{Introduction}

During the last decade, many studies have explored the possibility to use sunlight as a renewable energy source by using artificial molecular networks able to mimic natural photosynthesis. ${ }^{[1,2]}$ These networks can be obtained from the combination of different moieties with distinct functionalities in order to produce composite materials possessing suitable functions. In this scenario the new allotropic forms of carbon, like fullerenes, nanotubes and graphene showed interesting electronic and chemical properties that can be tuned by proper functionalization with targeted molecules. ${ }^{[3]}$ The modification of carbon structures with active molecules is the main step for obtaining hybrid nanomaterials with potential technological applications like sensors, in photovoltaic cells, in polymer composites and as catalysts, ${ }^{[4]}$ supercapacitors and other technologically relevant materials. ${ }^{[5-7]} A$ very interesting route to obtaining new carbon-based materials is through chemical functionalization by the Prato and Maggini reaction. ${ }^{[8]}$

[a] Dr. F. Possanza, Dr. F. Limosani, Prof. P. Tagliatesta Dipartimento di Chimica

Università degli Studi di Roma "Tor Vergata"

Via della Ricerca Scientifica 1, 00133-Rome, Italy

E-mail: pietro.tagliatesta@uniroma2.it

[b] Prof. R. Zanoni

Dipartimento di Chimica

Università degli Studi di Roma "La Sapienza"

Piazzale Aldo Moro 5, 00185-Rome, Italy

E-mail: robertino.zanoni@uniroma1.it

[c] Dr. M. Scarselli

Dipartimento di Fisica

Università degli Studi di Roma "Tor Vergata"

Via della Ricerca Scientifica 1, 00133-Rome, Italy

[d] Dr. E. Ciotta, Prof. R. Pizzoferrato

Dipartimento di Ingegneria Industriale Università degli Studi di Roma "Tor Vergata" Via del Politecnico 1, 00133-Rome, Italy

Supporting information for this article is available on the WWW under https://doi.org/10.1002/cphc.201800277
Such reaction has been extensively applied to fullerenes (Figure 1a), as also reported in the literature for the synthesis of

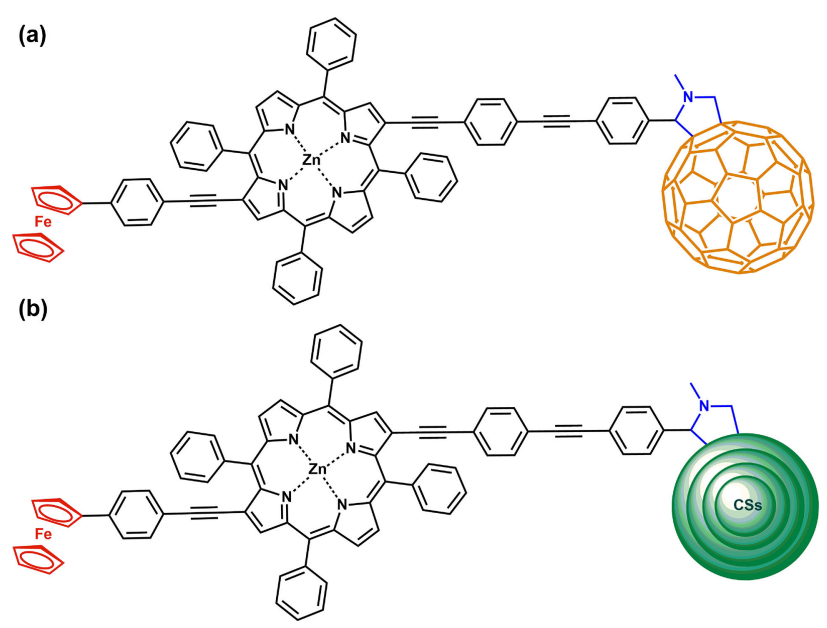

Figure 1. Structure of triads: (a) Ferrocene-Porphyrin-Fullerene (Triad 7). (b) Ferrocene- Porphyrin-Carbon Spheres (Triad 6).

several photoactive compounds. ${ }^{[9-11]}$ In the last twenty years porphyrins, C60 and ferrocene-containing dyads, triads and tetrads have been synthesized and variously assembled and the resulting species have been studied for mimicking natural photosynthetic systems. ${ }^{[12-14]}$ In the field of solar energy conversion, many recently published papers mainly focused on fullerenes applied to organic solar cells operating in solution and on carbon nanotubes in the solid state. In the present study, we investigated the functionalization of carbon spheres (CSs), a material recently produced in our laboratories. ${ }^{[15]}$ The functionalization was carried out with a dyad built by interconnecting a porphyrin and a ferrocene with a triple bond, as shown in Figure 1b. Different experimental techniques have 
been used to characterize the structure and properties of this new carbon-based material. The results indicate that the adduct shows good stability and optical performance.

\section{Results and Discussion}

Recently the beta functionalization of $\mathrm{H}_{2}$ TPP by one or two ferrocene molecules in the 2 and 3 positions through ethynyl or phenylethynyl groups was reported by some of us. ${ }^{[16]}$ Two new triads, connecting ferrocene and $\mathrm{C} 60$ to the 2,12 pyrrole positions of $\mathrm{H}_{2}$ TPP through ethynyl bonds were obtained and used as models to further investigate their electron-transfer properties. ${ }^{[17]}$ Reports from other groups have followed on the synthesis and characterization of different tetraphenylporphyrins bearing ferrocene at beta, meso or 4-phenyl positions. ${ }^{[11,18,19]}$
The correct stereochemistry of 2,12-dibromo TPP starting compound was recently elucidated. ${ }^{[20]}$ In Scheme 1 the synthetic steps for obtaining the CSs and C60 triads are reported.

The first step involved Sonogashira coupling of compound $1^{[21]}$ with 1.5 equivalents of 4-[(4'-ethynyl)phenyl]-ethynylbenzaldehyde, 2, giving the porphyrin derivative 3 in $36 \%$ yield after chromatographic purification. The following step was again a Sonogashira coupling reaction between compound 3 and 2 equivalents of 4-ferrocenylphenylacetylene, giving the intermediate 4 in $72 \%$ yield. Zinc was inserted following a literature procedure to quantitatively give Dyad 5. Finally, Dyad 5 was used to obtain Triad 6.

The CSs starting material was obtained by a CVD process and a detailed characterization of the outer and inner structure of the spheres as well as their spectroscopic properties will be reported in detail elsewhere and is here summarized. ${ }^{[15]}$

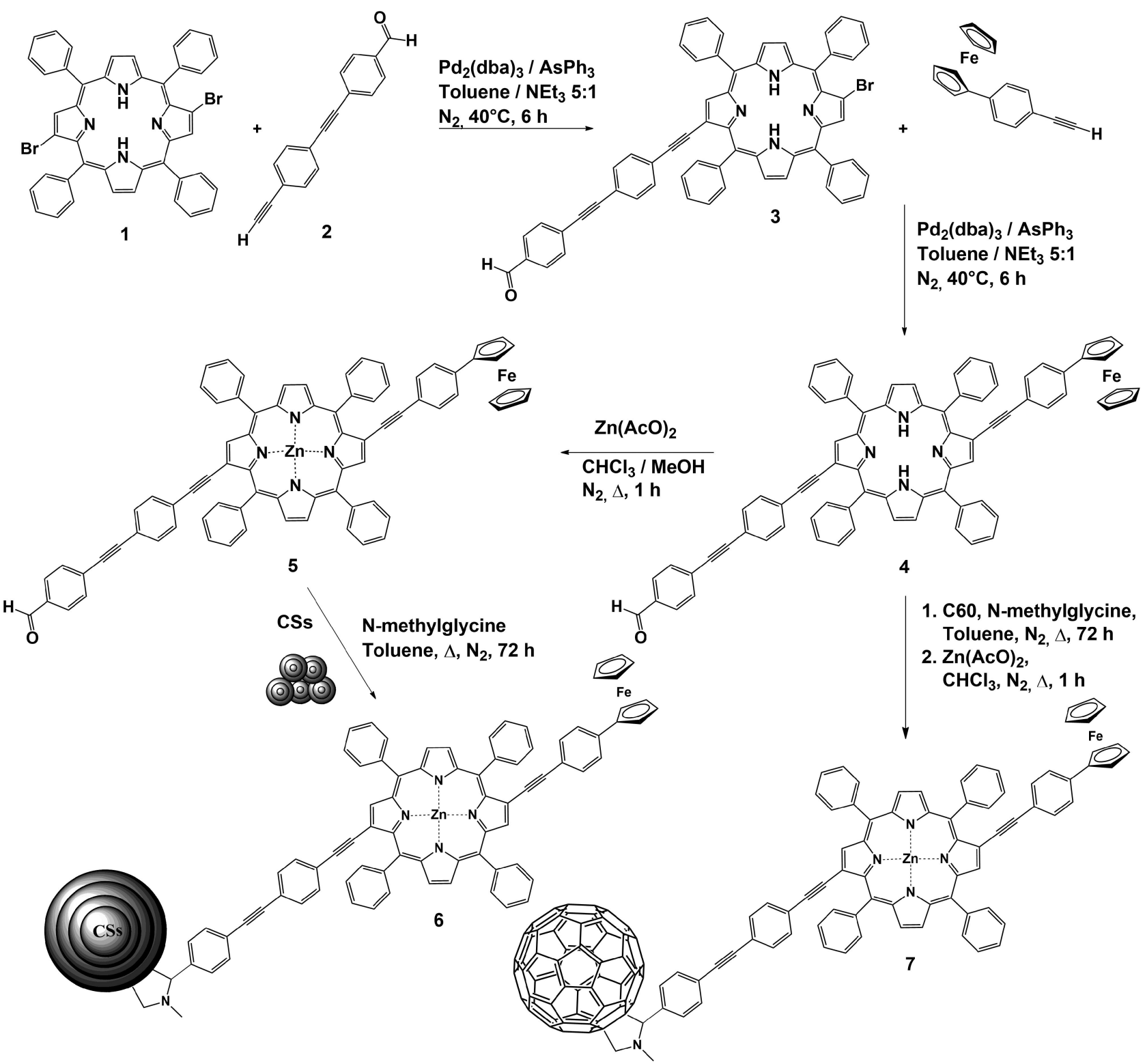

Scheme 1. Synthetic pathways for obtaining the CSs and C60 Triads. 
Figure 2 reports a SEM micrograph that shows the presence of spheres of variable size, some of which partially fused

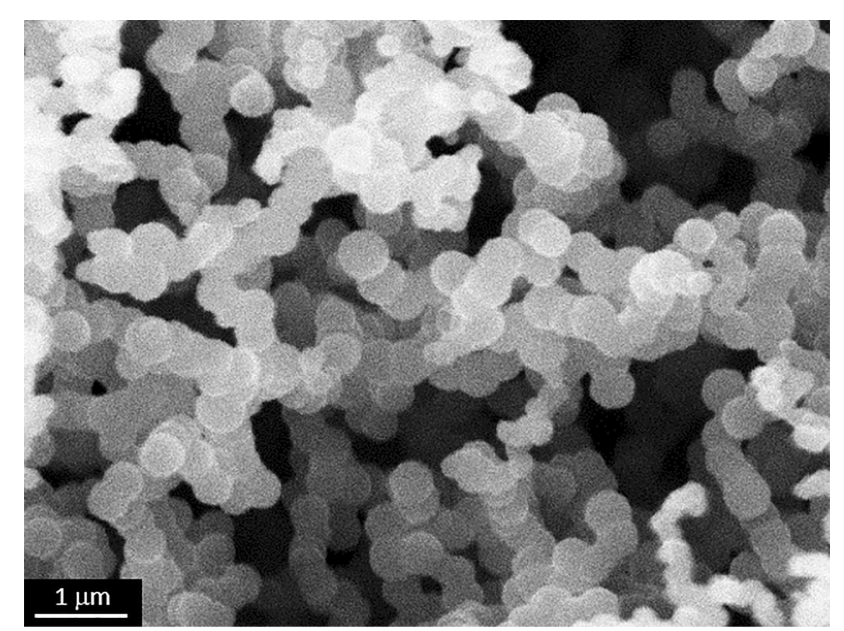

Figure 2. The SEM picture of the carbon spheres.

together. No side products were found on the investigated different sample areas.

TEM helped investigating the internal structure of the samples and their size distribution. Figure 3 reports a low

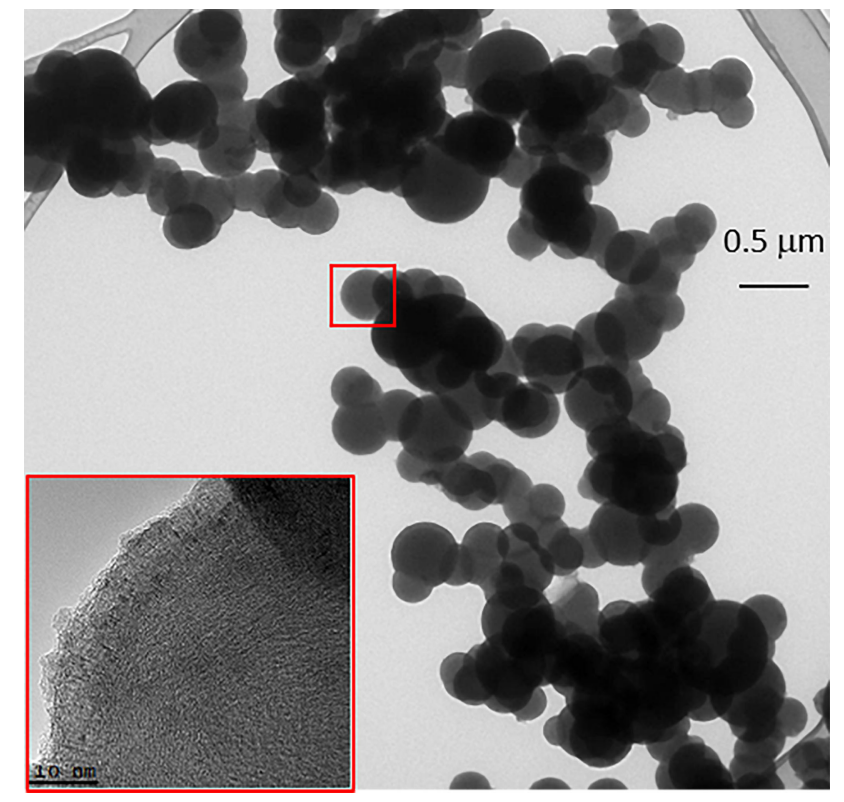

Figure 3. The TEM picture of the carbon spheres.

magnification image of many spheres dispersed on the TEM grid.

The average CSs diameter makes the TEM analysis rather difficult. In addition, the high magnification image of a single sphere reported in the inset, shows ordered graphitic layers in the region close to the surface that spans around $10-15 \mathrm{~nm}$ range. This region defines the spatial extension of those planes nearly parallel oriented to the electron beam. The inner layers look less ordered, although they still have a concentric arrangement. The average CSs diameter found from a statistical analysis performed on several images of comparable spatial resolution is $175 \pm 10 \mathrm{~nm}$.

XPS analysis was performed on the CSs after functionalization process in order to investigate the attachment of the dyad on the surface of the CSs and the resulting chemical composition. Figure 4 shows the Fe $2 p_{3 / 2,1 / 2}$ and $N$ 1s photo-

\section{(a)}

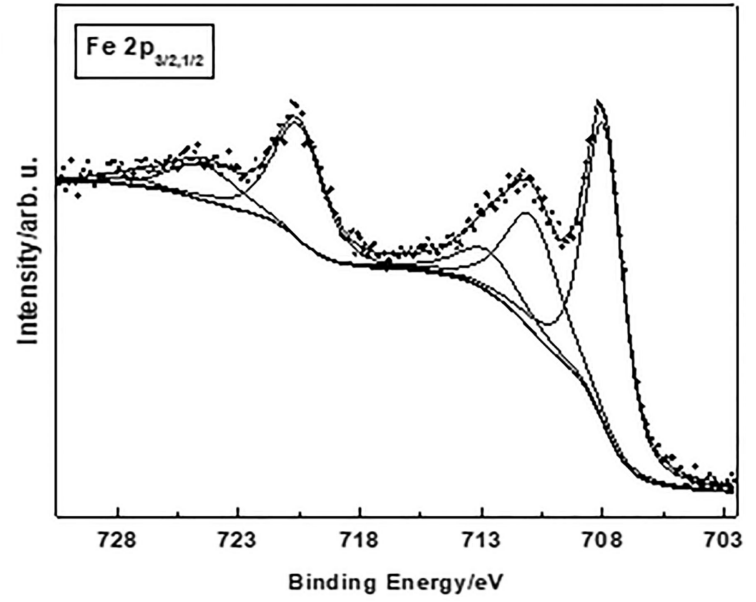

(b)

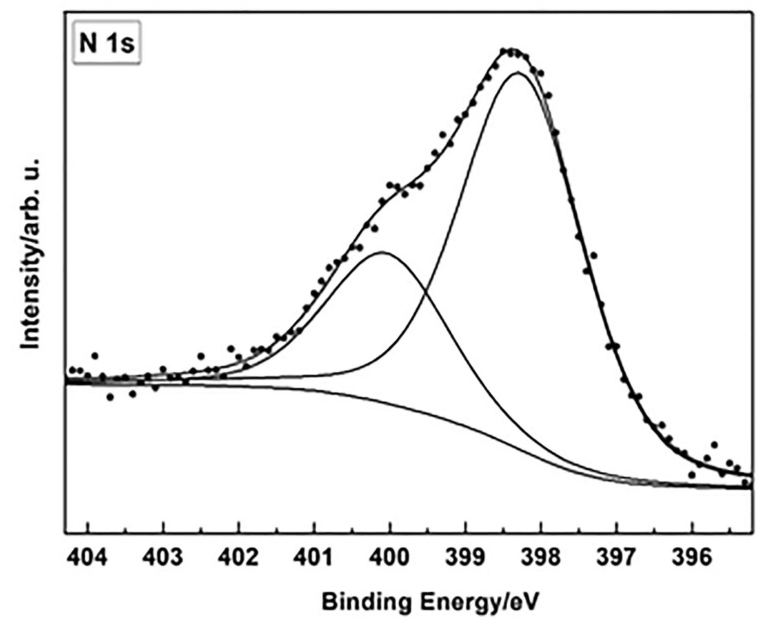

Figure 4. Fe $2 \mathrm{p}_{3 / 2,1 / 2}$ (a) and $\mathrm{N} 1 \mathrm{~s}$ (b) photoemission regions of Triad 6. Experimental curve (dots) and peak-fit components (lines) are shown together.

emission region of the dyad/CSs sample. The Fe $2 p$ region presents two major peaks at $708.0\left(2 \mathrm{p}_{3 / 2}\right)$ and $720.5\left(2 \mathrm{p}_{1 / 2}\right) \mathrm{eV}$, plus a second couple of Fe $2 p$ signals, falling at $710.9\left(2 p_{3 / 2}\right)$ and $724.4\left(2 \mathrm{p}_{1 / 2}\right) \mathrm{eV}$.

A satellite line at higher energy accompanies each of the latter two contributions. The $\mathrm{Fe}(\mathrm{II}) / \mathrm{Fe}$ (III) atomic ratio is 1.4 . On the basis of the literature, ${ }^{[2]}$ such complex envelope of peaks corresponds to the presence of both $\mathrm{Fe}$ (II) and $\mathrm{Fe}$ (III) ionic states, which we attribute to the interaction of ferrocene valence orbitals with the conjugated network of $s p^{2}$ hybridized carbons in the CSs. 
In fact, in some previous literature reports by some of us on redox-active Si (100) covalently functionalized surfaces with [60] fullerene conjugates, ${ }^{[23]}$ the XPS spectra from Fe $2 p$ revealed the presence of $\mathrm{Fe}(\mathrm{III})$ and $\mathrm{Fe}(\mathrm{II})$ states in a different ratio with respect to substituted ferrocene moieties directly bound to the same Si surface via an anchoring $\mathrm{C}=\mathrm{C}$ or $\mathrm{C} \equiv \mathrm{C}$ arm. This result was taken as an evidence of the electron-withdrawing effect of fullerene compared to Si. Given the present results, a similar electronic effect can be assigned to the CSs, which calls for an energy levels relative alignment between the dyad and the CSs allowing partial electron donation from the ferrocene moiety to the CSs.

The $\mathrm{N}$ 1s peak presents two distinct components which we assign to porphyrinate nitrogens $(398.3 \mathrm{eV})$ and substituted amine groups $(400.1 \mathrm{eV})$, in a 2.5 atomic ratio. Such co-presence was expected because of the anchoring bond of the dyad to the CSs and confirms the nature of the bonding. The atomic ratio is somehow lower than expected and we assign it to some residual aminic contamination in the final sample. The $\mathrm{Zn} / \mathrm{Fe}$ atomic ratio is equal to 1 , confirming the intact nature of the attached dyad, as shown in Figure 4.

The graphitic nature of the CSs was investigated using Raman Spectroscopy, a powerful and popular tool for the investigation of the extent of graphitization in carbon nanomaterials. The Raman shifts which appear at $\sim 1360 \mathrm{~cm}^{-1}$ and $\sim 1600 \mathrm{~cm}^{-1}$ in Figure 5 are generally denoted as D-band and G-

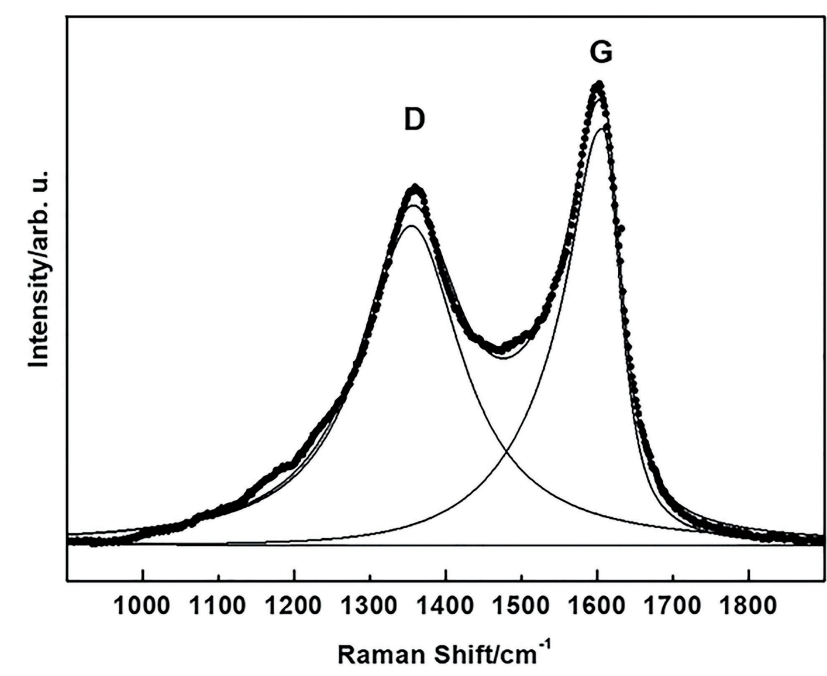

Figure 5. Raman spectrum of the functionalized spheres of Triad 6.

band, respectively. The D-band arises from aromatic rings but needs defects to be activated, as it is associated with dangling bonds for the in-plane termination of disordered graphite. The G-band corresponds to the E2g phonon of the in-plane vibration of $s p^{2}$-bonded carbon atom, is related to the vibration of $s p^{2}$-bonded carbon atom in a two-dimensional hexagonal lattice and represents the extent of graphitization. The structural quality of CSs was analyzed from the $I_{D} / I_{G}$ ratio, ${ }^{[24-26]}$ a parameter widely used as a measure of the size of the $s p^{2}$ ring clusters in a network of $s p^{3}$ - and $s p^{2}$-bonded carbons, therefore taken as an indication of structural distortions. The value of $I_{D} / I_{G}$ area ratio (1.3), obtained from curve-fitting, can be associated to a value of $L_{a}^{[27]}$ equal to $\sim 10 \mathrm{~nm}$, which consistently compares with the results from TEM reported in Figure 3. The D peak width can be related to the $C s p^{2}$ content because of its relationship with the fraction of carbon atoms not $s p^{2}$ hybridized. The CSs show quite large FWHM values (140$160 \mathrm{~cm}^{-1}$ ), indicating a low degree of crystalline perfection.

A comparison of the UV-vis absorption spectra of the new triads with those of ZnTPP reference compound (see Figure 6)

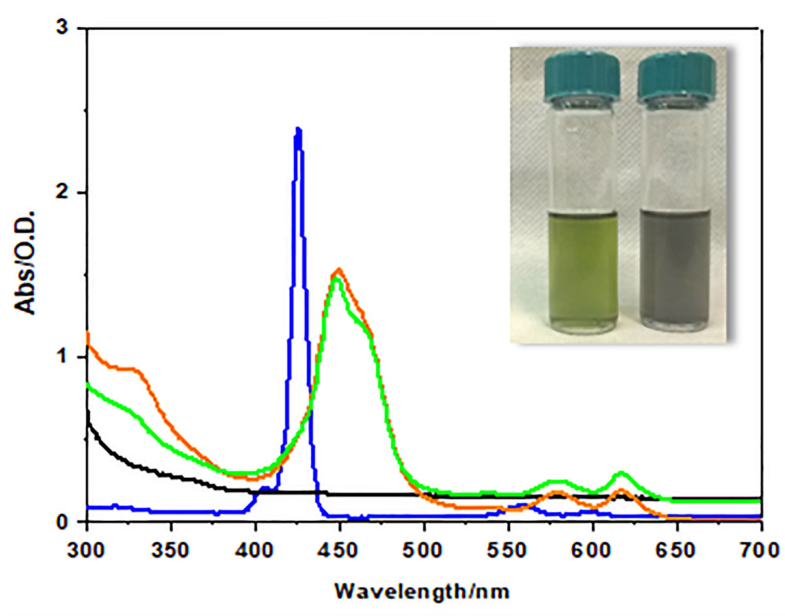

Figure 6. UV-vis absorption spectra of Triads 6 (green) and 7 (orange) and the reference compounds ZnTPP (blue) and CSs (black) in diluted DMF solutions. The insert shows the photographic image of the samples dispersed in DMF (from left to right: Triad 6 and only CSs).

shows that the new adducts exhibit an appreciable broadening of the Soret band and a significant red shift $(\sim 20 \mathrm{~nm})$ of both the Soret and the $\mathrm{Q}$ bands. This suggests that conjugation is more extended in the triads, and the porphyrin HOMO spreads more along the whole length of the linker. In this respect, the behaviour of the new triad compounds is distinct from that of the previouly reported ones ${ }^{[28]}$ and more similar to those recently investigated by our group. ${ }^{[21]}$ This confirms that the ferrocene unit favours delocalization of charge along the carbon-carbon triple bonds.

Figure 7 displays the PL emission spectra of the Dyad 5 (magenta curve), Triad 6 (green), Triad 7 (orange) where the dyad is bound to CSs and C60, respectively, and CSs (black). The emission spectrum of ZnTPP reference compound (blue curve) is also shown for comparison. The excitation wavelength was $\lambda_{\text {exc }}=435 \mathrm{~nm}$ and all the spectra were normalized to the optical density at the excitation wavelength, so they can be compared to each other, and the reported PL intensity is an estimate of the relative quantum efficiency. First, the comparison shows that the spectral distribution of the emitted energy in all the adducts closely resembles the singlet excited-state emissions of ZnTPP reference compound, apart from a red shift of $\sim 25 \mathrm{~nm}$ (see Table 1) which confirms the more extended conjugation discussed above on the basis of the UV-vis absorption spectra. 


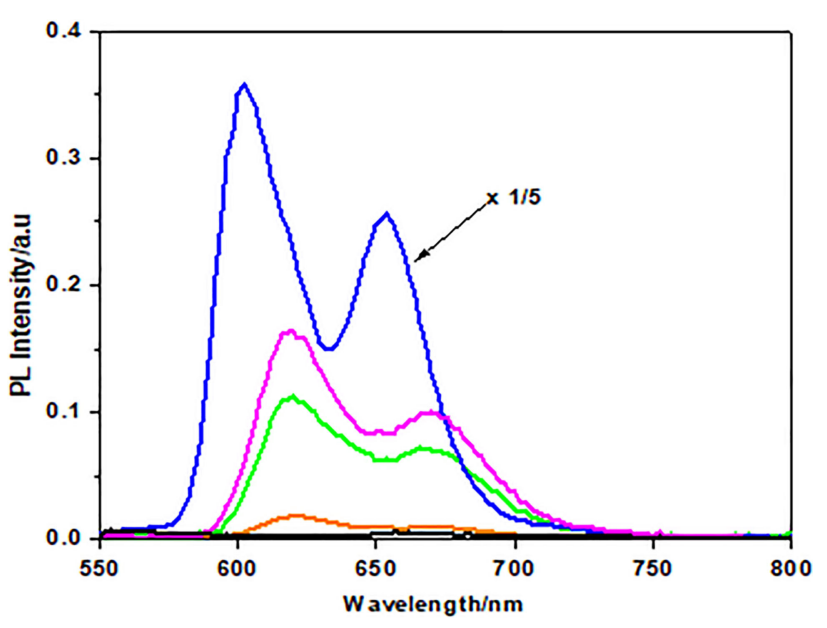

Figure 7. Photoluminescence spectra of ZnTPP (blue), Dyad 5 (magenta), Triads 6 (green), 7 (orange) and CSs (black) in diluted DMF solutions.

Table 1. Photoluminescence parameters of the investigated compounds in DMF: the wavelength of the emission peaks and the PL quantum yield (QY)

\begin{tabular}{llll} 
Adduct & $\mathrm{PL} \lambda_{\max 1}[\mathrm{~nm}]$ & $\mathrm{PL} \lambda_{\max }[\mathrm{nm}]$ & $\mathrm{QY}$ \\
\hline Dyad 5 & 620 & 670 & $1.5 \times 10^{-3}$ \\
Triad 6 & 620 & 668 & $1.1 \times 10^{-3}$ \\
Triad 7 & 620 & 670 & $1.6 \times 10^{-4}$ \\
\hline
\end{tabular}

Second, the quantum efficiency of the dyad decreases only by $\sim 30 \%$ when bound to CSs in 6 , indicating a moderate interaction with CSs. A significant PL quenching of about $90 \%$ is instead observed when the dyad is linked to $\mathrm{C} 60$ in 7 , in agreement with the reported quenching behaviour of $\mathrm{C} 60$, due to its tendency to form charge-trasfer complexes with electron donating groups. ${ }^{[29]}$ Since similar strong quenching effects on covalently bound fluorophores are also produced by graphene ${ }^{[30]}$ and, single-walled carbon nanotubes, ${ }^{[31]}$ CSs are distinct from other carbon-based materials because of their ability to bind optical emitting molecules without strongly affecting their quantum yield, a promising property for optoelectronic ${ }^{[32]}$ and bioimaging applications.

\section{Conclusions}

In this paper, we have demonstrated that CSs can be covalently functionalized with photoactive molecules with a resulting push-pull effect generated by the ferrocene and carbon moieties. CSs modified with metal porphyrin have been prepared by an exact synthetic route; each intermediate compound that was synthesized to arrive at the final product was characterized using the most common techniques, mass spectrometry and NMR analysis. The functionalization method here proposed is very efficient and gives as a result a new carbon material with spectroscopic characteristics similar to what already reported for fullerene derivatives. The structural properties of new hybrid were characterized by using spectroscopic techniques. Photophysical studies showed that this compound may be a promising candidate for optoelectronic applications. Such assembly can be considered as a new photoactive material and its possible uses are for building cells able to generate current and/or in sensors.

\section{Experimental Section}

\section{General Methods}

${ }^{1} \mathrm{H}$ NMR spectra was recorded as $\mathrm{C}_{6} \mathrm{D}_{6}$ solution on a Bruker $\mathrm{AM}-300$ instrument using residual solvent signal as an internal standard (7.15 for $C_{6} D_{6}$ ). Chemical shifts are given as $\delta$ values. J values are given in $\mathrm{Hz}$. FAB (Fast Atomic Bombardment) mass spectra were measured on a VG-4 spectrometer using m-nitrobenzyl alcohol (NBA) as a matrix. UV-vis spectra were acquired by a Varian Cary 50 Scan spectrophotometer in DMF or $\mathrm{CH}_{2} \mathrm{Cl}_{2}$ solution. Photoluminescence (PL) emission spectra were obtained by using a specific laboratory set-up ${ }^{[33]}$ equipped with a continuous-wave 200-W $\mathrm{Hg}(\mathrm{Xe})$ discharge lamp (Oriel Corp.) monochromatized with an excitation $25-\mathrm{cm}$ monochromator (PMI), an emission $25-\mathrm{cm}$ monochromator (Oriel Cornerstone 260) and a Hamamatsu R3896 photomultiplier as emission light detector. Diluted solution in spectroscopic DMF, with an absorbance $\angle 0.1$ OD in the range 250$400 \mathrm{~nm}$, were prepared for the PL spectra and characterized by using conventional $90^{\circ}$ geometry on fused-silica cuvettes with an optical path-length of $10 \mathrm{~mm}$. The typical excitation wavelength of $\lambda_{\text {exc }}=435 \mathrm{~nm}$ was used with a spectral band-pass of $3 \mathrm{~nm}$ for both the excitations and emission monochromators. The curves were corrected for the spectral response of the set-up that was previously calibrated in the spectral range from $300 \mathrm{~nm}$ to $800 \mathrm{~nm}$ by using a reference black-body lamp and standard-fluorophore solutions. A spectral band-pass of $3 \mathrm{~nm}$ was used for both the excitations and emission monochromators. X-ray Photoelectron Spectroscopy (XPS) measurements were obtained from a modified Omicron NanoTechnology MXPS system. The spectra were excited by achromatic $\mathrm{Mg} \mathrm{K \alpha}$ and $\mathrm{Al} \mathrm{K \alpha}$ photons $(h v=1253.6$ and $1486.6 \mathrm{eV}$, respectively), generated operating the anode at 14$15 \mathrm{kV}, 10-20 \mathrm{~mA}$. All the samples were mounted on metal tips as thin layers of pressed powders. Experimental spectra were theoretically reconstructed by fitting the peaks to symmetric pseudo-Voigt functions (linear combination of gaussian and lorentzian peaks) and the background to a Shirley or a linear function. XPS atomic ratios ( $\pm 10 \%$ associated error) between relevant core lines were estimated from experimentally determined area ratios corrected for the corresponding theoretical cross sections ${ }^{[34]}$ and for a square root dependence of the photoelectrons kinetic energies. All binding energies were referenced to the lowest lying $C$ 1s peak component, taken at $285.0 \mathrm{eV}$, which was assumed to be related to the aromatic $\mathrm{C}$ atoms. Raman spectra were acquired at room temperature in backscattering geometry with an inVia Renishaw micro-Raman spectrometer equipped with an air-cooled CCD detector and super-Notch filters. The emission line $\lambda$ laser at $488.0 \mathrm{~nm}$ from an $\mathrm{Ar}^{+}$ion laser was focused on the sample under a Leica DLML microscope using a $5 \times$ objective. The power of the incident beam was about $5 \mathrm{~mW} .10 \mathrm{~s}$ accumulations were generally acquired for each sample. The resolution was $2 \mathrm{~cm}^{-1}$, and spectra were calibrated using the $520.5 \mathrm{~cm}^{-1}$ line of a silicon wafer. $L_{a}$ (the average crystallite size) of the $s p^{2}$ lattice was calculated using the equation: $L_{a}=13.611\left(I_{D} / I_{G}\right)^{-1},{ }^{[35]}$ for the present value of $\lambda$ laser, where $I_{G}$ and $I_{D}$ are the intensities of the Raman $G$ and $D$ bands, respectively.

These last were obtained by a curve-fit procedure employing a pseudo-Voigt profile (linear combination of gaussian and lorentzian curves) and an asymmetric pseudo-Voigt profile, respectively. The 
SEM micrographs were acquired using a field emission gun scanning electron microscopy (FEG-SEM, Leo Supra 35), and a transmission electron microscopy (TEM, cold FEG Hitachi HF2000 operated at $200 \mathrm{kV}$ voltage). For the TEM analysis, the spheres were dispersed in ethanol ( $95 \%$ solution) and sonicated for half an hour. The samples were then dropped onto a copper grid (mesh 300) covered with a thin holey carbon film and dried before investigation.

\section{Chemicals}

Silica gel 60 (70-230 and 230-400 mesh, Merck) was used for column chromatography. High-purity-grade nitrogen gas was obtained from Rivoira. All the reagents and solvents were from Fluka Chem. Co., Aldrich Chem. Co., or Carlo Erba and were used as received. 4-ferrocenylphenylacetylene was synthesized as reported in the literature. ${ }^{[27]}$

\section{Synthesis of Carbon Spheres}

CSs were obtained following a chemical vapor deposition (CVD) process carried out in a horizontal hot-wall quartz furnace. The furnace was heated up to the growth temperature under argon atmosphere at ambient inert pressure (760 Torr). Ferrocene was used as catalyst and dissolved in o-dichlorobenzene. The solution was placed in a $10 \mathrm{~mL}$ glass syringe and injected into the growth chamber at a constant rate of $6 \mathrm{mLh}^{-1}$ through a flux of argon and acetylene $(250 / 45 \mathrm{sccm})$ which act as gas carrier and carbon precursor, respectively. The vaporized solution and the gas mixture were admitted in the chamber through a small stainless-steel capillary, which enables the vaporized solution to be injected directly into the high temperature region of the quartz tube furnace. The CSs synthesis was carried out at $800-900^{\circ} \mathrm{C}$, the temperature being checked by an optical infrared pyrometer and the synthesis lasted about 2 hours. The final powdered product was collected from the quartz tube, characterized, and used without any cleaning process.

\section{Synthesis of Porphyrins Linked to Carbon Spheres}

Porphyrin-ferrocene compound $\mathbf{4}$ was synthesized as reported in the literature. ${ }^{[28]}$

Dyad $5.60 \mathrm{mg}(0.058 \mathrm{mmol})$ of compound 4 were dissolved in $30 \mathrm{~mL}$ of $\mathrm{CHCl}_{3}$ and $1 \mathrm{~mL}$ of saturated solution of $\mathrm{Zn}(\mathrm{AcO})_{2}$ in methanol was added. The mixture was refluxed and stirred for $1 \mathrm{~h}$. The solvent was removed under vacuum and the crude product was purified by a plug of silica gel eluted with chloroform. The fraction containing the desired product was collected and the solvent was evaporated. The compound was crystallized from dichloromethane/methanol. Dyad 5 (68 mg, 99\%).

MS (FAB): $m / z\left[M+H^{+}\right]=1189$ (see Fig. S1); ${ }^{1} \mathrm{H}$ NMR: $(300 \mathrm{MHz}$, $\left.\mathrm{C}_{6} \mathrm{D}_{6}\right) 9.59(\mathrm{~s}, 1 \mathrm{H}), 9.57(\mathrm{~s}, 1 \mathrm{H}), 9.44(\mathrm{~s}, 1 \mathrm{H}), 9.05-8.78(\mathrm{~m}, 4 \mathrm{H})$, 8.43-8.11 (m, $9 \mathrm{H}), 7.64-7.43(\mathrm{br} m, 15 \mathrm{H}), 7.39(\mathrm{~d}, 2 \mathrm{H}, \mathrm{J}=8.0 \mathrm{~Hz})$, $7.34(\mathrm{~d}, 2 \mathrm{H}, \mathrm{J}=8.1 \mathrm{~Hz}), 7.30(\mathrm{~s}, 4 \mathrm{H}), 4.49(\mathrm{~s}, 2 \mathrm{H}), 4.10(\mathrm{~s}, 2 \mathrm{H}), 3.92$ $\left(\mathrm{s}, 5 \mathrm{H}\right.$ ) (see Fig. S2); UV/Vis $\lambda_{\max }\left(\mathrm{CH}_{2} \mathrm{Cl}_{2}\right) / \mathrm{nm} 445$ (log $\left.\varepsilon 4.85\right), 571$ (3.95), 611 (3.99) (see Fig. S3).

Triad $6.20 \mathrm{mg}(0.0168 \mathrm{mmol})$ of Dyad $5,40 \mathrm{mg}$ of CSs and $30 \mathrm{mg}$ $(0.34 \mathrm{mmol}) \mathrm{N}$-methylglycine were refluxed for $72 \mathrm{~h}$ in $20 \mathrm{~mL}$ of anhydrous toluene under $\mathrm{N}_{2}$ atmosphere. The obtained black solid was washed with warm toluene and filtered until the solvent was uncoloured. The final compound was dried at $60^{\circ} \mathrm{C}$ under vacuum for 10 hours.
UV/Vis $\lambda_{\max }(\mathrm{DMF}) / \mathrm{nm} 302,448,580,618$.

\section{Acknowledgements}

We thank Prof. I. Cacciotti from Dipartimento di Ingegneria, Università di Roma Niccolò Cusano (INSTM-UdR), Italy for the SEM measurements and E. Gautron from Institut des Matériaux Jean Rouxel (IMN) Université de Nantes, France, for the TEM measurements. M. Scarselli acknowledges European Community for the RISE Project CoExAN GA644076.

\section{Conflict of Interest}

The authors declare no conflict of interest.

Keywords: carbon materials • ferrocene • fluorescence porphyrins $\cdot$ Raman spectroscopy

[1] D. M. Guldi, M. Prato, Acc. Chem. Res. 2000, 33, 695-703.

[2] S. Fukuzumi, D. M. Guldi in Electron Transfer in Chemistry (Eds.: V. Balzani), Wiley-VCH, Weinheim, 2001, pp. 270-326.

[3] A. Krueger in Carbon Materials and Nanotechnology, Wiley-VCH Verlag GmbH \& Co. KGaA, Weinheim, 2010.

[4] R. Hoffmann, A. Kabanov, A. Golov, D. Proserpio, Angew. Chem. Int. Ed. 2016, 55, 10962-10966; Angew. Chem. 2016, 128, 11122-11139.

[5] D. Kiessling, R. D. Costa, G. Katsukis, J. Malig, F. Lodermeyer, S. Feihl, A. Roth, L. Wibmer, M. Kehrer, M. Volland, P. Wagner, G. G. Wallace, D. L. Officer, D. M. Guldi, Chem. Sci. 2013, 4, 3085-3098.

[6] M. B. M. Krishna, V. P. Kumar, N. Venkatramaiah, R. Venkatesan, D. N. Rao, Appl. Phys. Lett. 2011, 98, 081106.

[7] D. Jariwala, V. K. Sangwan, L. J. Lauhon, T. J. Marks, M. C. Hersam, Chem. Soc. Rev. 2013, 42, 2824-2860.

[8] M. Maggini, G. Scorrano, M. Prato, J. Am. Chem. Soc. 1993, 115, 97989799.

[9] D. M. Guldi, N. Martin in Fullerenes: From Synthesis to Optoelectronic Properties, Kluwer Academic Publishers, Springer Netherlands, 2002.

[10] H. Imahori, K. Tamaki, D. M. Guldi, C. Luo, M. Fujitsuka, O. Ito, Y. Sakata, S. Fukuzumi, J. Am. Chem. Soc. 2001, 123, 2607-2617.

[11] M. U. Winters, E. Dahlstedt, H. E. Blades, C. J. Wilson, M. J. Frampton, H. L. Anderson, B. Albinsson, J. Am. Chem. Soc. 2007, 129, 4291-4297.

[12] P. Wurfel in Physics of Solar Cells in Basic Principles to Advanced Concepts, Wiley-VCH Verlag GmbH \& Co. KGaA, Weinheim, 2009.

[13] S. R. Wenham, M. A. Green, M. E. Watt, R. Corkish in Applied Photovoltaics, Earthscan Publications Ltd., London, 2006.

[14] M. Green in Third Generation Photovoltaics in Advanced Solar Energy Conversion, Springer Series in Photonics, Springer, Heidelberg, 2005.

[15] M. Scarselli, F. Limosani, M. Passacantando, F. D'Orazio, M. Nardone, I. Cacciotti, F. Arduini, E. Gautron, M. De Crescenzi, Adv. Mater. Interfaces. 2018, 1800070; DOI: 10.1002/admin.201800070.

[16] A. Lembo, P. Tagliatesta, D. M. Guldi, M. Wielopolski, M. Nuccetelli, J. Phys. Chem. A. 2009, 113, 1779-1793.

[17] P. Tagliatesta, R. Pizzoferrato, J. Organomet. Chem. 2015, 787, 27-32.

[18] A. Vecchi, E. Gatto, B. Floris, V. Conte, M. Venanzi, V. N. Nemykin, P. Galloni, Chem. Commun. 2012, 48, 5145-5147.

[19] V. N. Nemykin, G. T. Rohde, C. D. Barrett, R. G. Hadt, J. R. Sabin, G. Reina, P. Galloni, B. Floris, Inorg. Chem. 2010, 49, 7497-7509.

[20] G. Di Carlo, A. Orbelli Biroli, M. Pizzotti, F. Tessore, V. Trifiletti, R. Ruffo, A. Abbotto, A. Amat, F. De Angelis, P. R. Mussini, Chem. Eur. J. 2013, 19, 10723-10740.

[21] F. Limosani, F. Possanza, E. Ciotta, F. Pepi, C. Salvitti, P. Tagliatesta, R. Pizzoferrato, J. Porphyrins Phthalocyanines 2017, 21, 364-370.

[22] E. A. Dalchiele, A. Aurora, G. Bernardini, F. Cattaruzza, A. Flamini, P. Pallavicini, R. Zanoni, F. Decker, J. Electroanal. Chem. 2005, 579, 133142. 
[23] F. Cattaruzza, A. Llanes-Pallas, A. G. Marrani, E. A. Dalchiele, F. Decker, R. Zanoni, M. Prato, D. Bonifazi, J. Mater. Chem. 2008, 18, 1570-1581.

[24] A. C. Ferrari, J. Robertson, Phys. Rev. B. 2000, 61, 14095-14107.

[25] M. A. Pimenta, G. Dresselhaus, M. S. Dresselhaus, L. G. Cançado, A. Jorio, R. Saito, Phys. Chem. Chem. Phys. 2007, 9, 1276-1291.

[26] X. Diez-Betriu, S. Alvarez-Garcia, C. Botas, P. Alvarez, J. Sanchez-Marcos, C. Prieto, R. Menendez, A. de Andres, J. Mater. Chem. C. 2013, 1, 69056912.

[27] L. G. Cançado, K. Takai, T. Enoki, M. Endo, Y. A. Kim, H. Mizusaki, A. Jorio, L. N. Coelho, R. Magalhães-Paniago, M. A. Pimenta, Appl. Phys. Lett. 2006, 88, 163106.

[28] P. Tagliatesta, A. Lembo, A. Leoni, New J. Chem. 2013, 37, 3416-3419.

[29] K. Campbell, A. Zappas, U. Bunz, Y. S. Thio, D. G. Bucknall, J. Photochem. Photobiol. A. 2012, 249, 41-46.

[30] V. Georgakilas, M. Otyepka, A. B. Bourlinos, V. Chandra, N. Kim, K. C. Kemp, P. Hobza, R. Zboril, K. S. Kim, Chem. Rev. 2012, 112, 6156-6214.
[31] C. F. Chiu, N. Dementev, E. Borguet, J. Phys. Chem. A. 2011, 115, 95799584.

[32] V. Georgakilas, D. M. Guldi, R. Signorini, R. Bozio, M. Prato, J. Am. Chem. Soc. 2003, 125, 14268-14269.

[33] R. Pizzoferrato, T. Ziller, A. Micozzi, A. Ricci, C. Lo Sterzo, A. Ustione, C. Oliva, A. Cricenti, Chem. Phys. Lett. 2005, 414, 234-238.

[34] J. H. Scofield, J. Electron Spectrosc. Relat. Phenom. 1976, 8, 129-137.

[35] F. Tuinstra, J. L. Koenig, J. Chem. Phys. 1970, 53, 1126-1130.

Manuscript received: March 29, 2018

Accepted Article published: May 21, 2018

Version of record online: 


\section{ARTICLES}

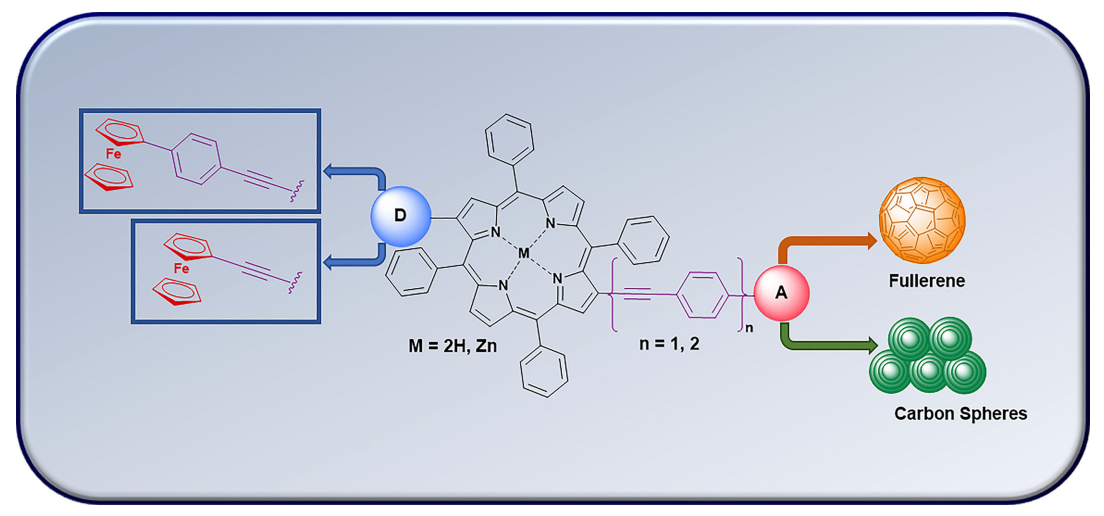

Dr. F. Possanza, Dr. F. Limosani, Prof. P. Tagliatesta*, Prof. R. Zanoni* Dr. M. Scarselli, Dr. E. Ciotta, Prof. R. Pizzoferrato

$1-8$

Functionalization of Carbon Spheres with a

Porphyrin-Ferrocene Dyad

Carbon Spheres were functionalized by metalloporphyrins and ferrocene bound each other by ethynyl or phenylethynyl groups. 